\title{
Fabrication and cold test of photonic band gap resonators and accelerator structures
}

\author{
Evgenya I. Smirnova, ${ }^{*}$ Ivan Mastovsky, Michael A. Shapiro, and Richard J. Temkin \\ Plasma Science and Fusion Center, Massachusetts Institute of Technology, 167 Albany Street, Cambridge, Massachusetts 02139, USA
}

Lawrence M. Earley and Randall L. Edwards

Los Alamos National Laboratory, P.O. Box 1663, Los Alamos, New Mexico 87544, USA

(Received 24 June 2005; published 20 September 2005)

\begin{abstract}
We present the detailed description of the successful design and cold test of photonic band gap (PBG) resonators and traveling-wave accelerator structures. Those tests provided the essential basis for later hot test demonstration of the first PBG accelerator structure at $17.140 \mathrm{GHz}$ [E. I. Smirnova, A. S. Kesar, I. Mastovsky, M. A. Shapiro, and R. J. Temkin, Phys. Rev. Lett., 95, 074801 (2005).]. The advantage of PBG resonators is that they were built to support only the main, $\mathrm{TM}_{01}$-like, accelerator mode while not confining the higher-order modes (HOM) or wakefields. The design of the PBG resonators was based on a triangular lattice of rods, with a missing rod at the center. Following theoretical analysis, the rod radius divided by the rod spacing was held to a value of about 0.15 to avoid supporting HOM. For a single-cell test the PBG structure was fabricated in X-band $(11 \mathrm{GHz})$ and brazed. The mode spectrum and $Q$ factor $(Q=5000)$ agreed well with theory. Excellent HOM suppression was evident from the cold test. A sixcell copper PBG accelerator traveling-wave structure with reduced long-range wakefields was designed and was built by electroforming at Ku-band $(17.140 \mathrm{GHz})$. The structure was tuned by etching the rods. Cold test of the structure yielded excellent agreement with the theoretical design. Successful results of the hot test of the structure demonstrating the acceleration of the electron beam were published in E.I. Smirnova, A. S. Kesar, I. Mastovsky, M. A. Shapiro, and R. J. Temkin, Phys. Rev. Lett., 95, 074801 (2005).
\end{abstract}

DOI: 10.1103/PhysRevSTAB.8.091302

\section{INTRODUCTION}

Two-dimensional (2D) metallic photonic band gap (PBG) structures [1] have received considerable attention recently, because of their possible applications in accelerators [2,3]. Future linear accelerator operating frequencies may increase from $S$-band to $X$ - and possibly to Ku-band or higher frequency band, because the higher frequency improves the energy efficiency of the accelerator. At high frequencies, it is crucial to use new accelerating cavities that suppress wakefields, because the excitation of wakefields intensifies with frequency and the wakefields induce transverse motion of electrons and emittance growth [4,5]. PBG structures may be applied for constructing the accelerating cavities with long-range wakefield suppression, based on their remarkable properties to reflect waves in certain ranges of frequencies (called "global band gaps") while allowing other frequencies to propagate. We experimentally demonstrate that the use of PBG structures is a promising approach to long-range wakefield suppression.

A PBG structure, or simply photonic crystal, represents a periodic lattice of macroscopic pieces, metallic, dielectric or both [6]. For accelerator applications, we employ a two-dimensional metallic photonic band gap structure, which is formed by a triangular array of copper rods [7]. We form a "PBG cavity" by removing a rod in the periodic structure (Fig. 1). The mode, which has a frequency in the band gap, will not be able to propagate out transversely

*Electronic address: smirnova@lanl.gov
PACS numbers: 29.17.+w, 41.75.Lx, 42.70.Qs, 84.40.-x

through the bulk of the PBG structure and will thus be localized inside the PBG cavity around the defect. First attempts to study 2D PBG resonators based on arrays of metal rods were made by the authors of [2,8]. Smith et al. [8] constructed and tested a metallic 2D PBG resonator. Mode confinement in this resonator was successfully proven. However, higher-order mode (HOM) suppression

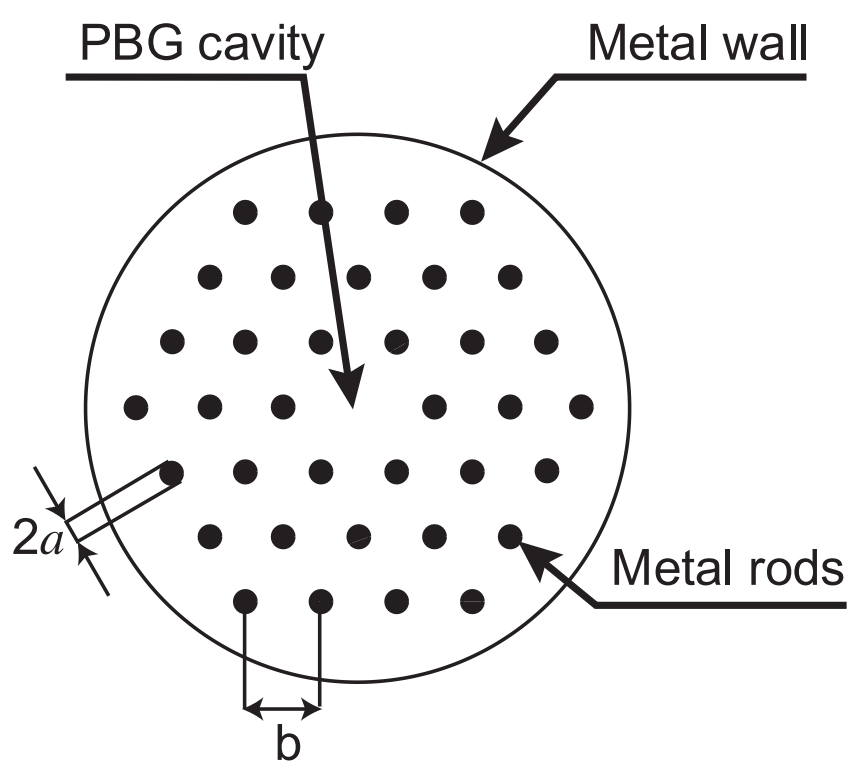

FIG. 1. The geometry of a PBG resonator formed by removing a single rod in a triangular array. 
in a metallic 2D PBG resonator was not proven in $[2,8]$, since the complete theory of global band gaps in 2D metallic PBG structures did not exist at that time. Global band gap diagrams for 2D metallic PBG structures were published in [7]. Based on the global band gap diagrams, we designed and constructed a single-mode 2D PBG resonator. The design and construction of a PBG accelerator with long-range wakefield suppression became possible after the success of a single-mode 2D PBG resonator demonstration. Finally, the PBG accelerator was successfully tested with the electron beam (hot tested) and the first ever demonstration of the acceleration in PBG structures was completed. The hot test results are published in [9]. The structure was powered with $2 \mathrm{MW}$ of microwaves and the electron beam was accelerated by $1.4 \mathrm{MeV}$. The accelerating gradient of $35 \mathrm{MV} / \mathrm{m}$ was demonstrated.

This paper provides a detailed description of the design, fabrication, and cold test of a series of photonic band gap (PBG) test resonators and finally of a six-cell, $17 \mathrm{GHz}$ PBG traveling-wave accelerator structure. The paper is organized as follows. In Sec. II, we derive the conditions for which a mode with the frequency inside the global band gap can be selectively confined inside the PBG cavity. Next, we design a single-mode 2D PBG resonator based on the global band gap picture from [7] and our computations using high frequency structure simulator (HFSS) code [10]. In Sec. III, we describe the construction and testing of several PBG resonators. We have demonstrated a high- $Q$ mode-selective PBG resonator at $11 \mathrm{GHz}$. Section IV presents the design of a 6-cell $2 \pi / 3$ traveling-wave (TW) PBG accelerator structure at 17.140 GHz. Section V describes the PBG accelerator structure manufacturing and tuning. The agreement between the design and final cold test results was excellent. Section VI is the conclusion.

\section{MODE CONFINEMENT IN 2D PBG RESONATORS}

We started the design of a single-mode PBG resonator by studying the global band gaps chart for the triangular lattice of metal rods, which was published in [7]. Electromagnetic waves with a frequency inside the band gap cannot propagate through the PBG structure and will be reflected from the array of elements, such as rods, which can be called the "PBG wall." However, by perturbing a single or several lattice sites, we can permit a single mode or set of closely spaced modes with frequencies inside the gap. The perturbation in two dimensions can be created by removing a single rod or replacing it with another one which has a size or shape different from the original. The wave with a frequency inside the band gap cannot propagate into the bulk of the PBG structure, so, under certain conditions, the mode of this frequency can be localized around the defect. Unlike in a traditional pillbox resonator only the modes with frequencies inside the band gap can be confined in a PBG cavity. Other frequencies will leak out through the PBG wall. Consequently, the spectrum of eigenmodes in a PBG resonator will be quite rare. The PBG resonator which can selectively confine an accelerating $\left(\mathrm{TM}_{01}\right.$-like) mode is a good candidate for an accelerator cell $[2,3]$.

We choose the simplest possible two-dimensional metallic PBG resonator, which is formed by one removed rod in a triangular lattice (Fig. 1). The rods are sandwiched between metal plates, which are so closely spaced that we do not have longitudinal (along the rods) field variation for the range of frequencies of interest. Analysis of the PBG resonators was performed using the HFSS code. We found that the mode with frequency in the band gap was confined in a PBG structure with only three rows of rods (Fig. 1). For this geometry, the diffraction $Q$ factor was found to be of the order of $10^{5}$, which is much higher than the computed Ohmic $Q$ factor of about 5000. Therefore, three rows of rods in this PBG structure are sufficient for construction of a PBG resonator.

We computed the eigenmodes of the PBG resonator of Fig. 1 for different ratios of the PBG structure rod radius, $a$, to the spacing between the rods, $b$. The computed eigenfrequencies for the TM modes are plotted in Fig. 2. The same band gap picture as in [7] is shown on the $\omega b / c$ versus $a / b$ diagram, where $\omega=2 \pi f, f$ is the wave frequency, $c$ is the speed of light. The solid black curve represents the boundary of the band gap region. A wave with a frequency to the right of the solid black curve cannot propagate through the PBG structure, while a wave with a frequency to the left of the black curve can propagate.

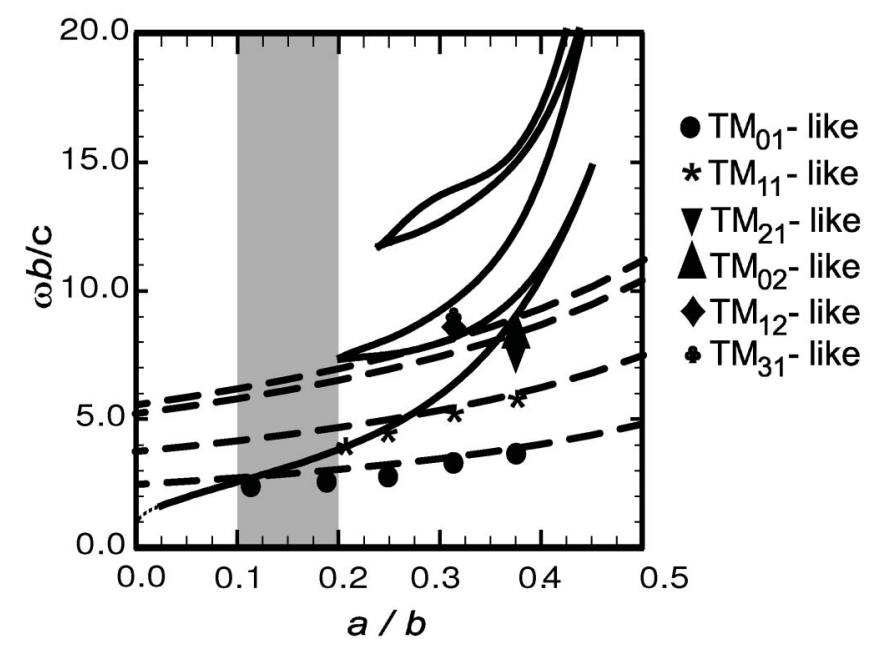

FIG. 2. The eigenfrequencies of the TM modes in PBG cavity formed by a single removed rod from the triangular lattice. The eigenfrequencies are plotted with dots for different ratios of $a / b$. Black solid lines show the band gap boundaries. Dashed lines represent the frequencies of modes of a pillbox cavity with the radius $R=b-a$. For the values of $a / b$ inside the shaded region the PBG resonator only confines a single, $\mathrm{TM}_{01}$-like mode. 
Modes with a frequency to the right of the curve are called "defect modes" and are confined in the defect region of the PBG structure, while modes to the left are not confined. The frequencies of the defect modes are plotted over the band gap picture with dots. The dashed lines on the picture show the eigenfrequencies of the pillbox cavity with a radius $R=b-a$. As the radius of the rods increases the effective radius of the PBG cavity goes down and the frequencies of the eigenmodes increase.

The field pattern of the modes confined inside the PBG resonators is shown in Fig. 3 [9]. We note from Fig. 2 that for some values of the radius of the rods $(0.1<a / b<0.2)$ only a single lowest order mode is confined by the PBG structure. This frequency range is shaded in Fig. 2. The field pattern of the confined mode resembles the pattern of the $\mathrm{TM}_{01}$ mode in a pillbox resonator [Fig. 3(a)], thus we named it the $\mathrm{TM}_{01}$-like mode. The $\mathrm{TM}_{01}$-like mode can be employed as an accelerating mode in a PBG accelerator cell. For this choice of $a / b$, the dipole mode, which is the $\mathrm{TM}_{11}$-like mode, is not confined within the central region or defect region of the cavity. However, the mode can still exist in the structure if a metal wall is placed at the periphery, as can be seen in Fig. 3(a). Therefore, for effective HOM damping, the outside wall should either
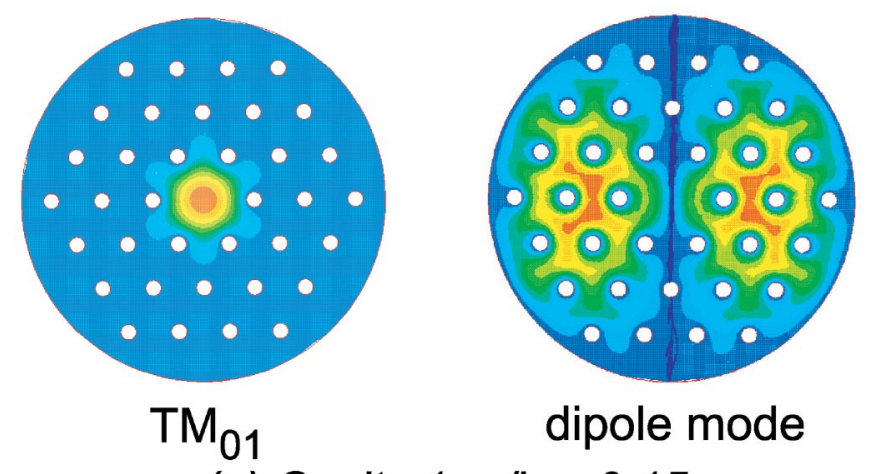

(a) Cavity $1, a / b=0.15$
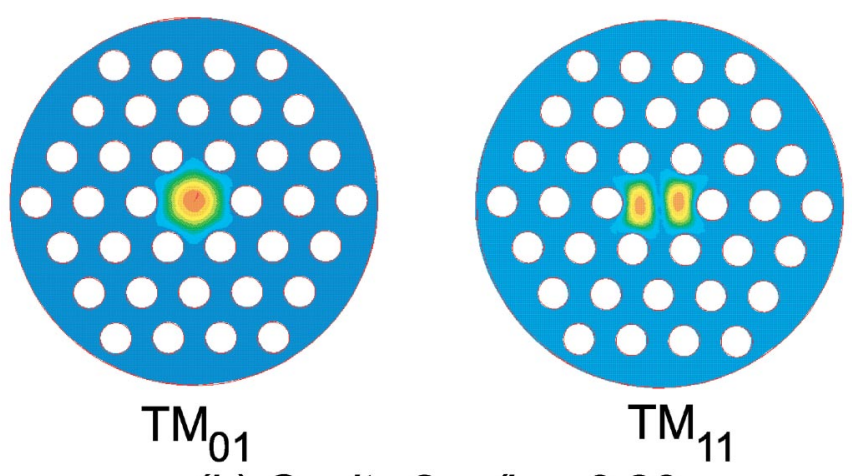

(b) Cavity 2, $a / b=0.30$

FIG. 3. (Color) Modes of PBG resonators with different ratios of $a / b$ : (a) $a / b=0.15$, the $\mathrm{TM}_{01}$ mode is confined by the PBG structure, a dipole mode is confined by the outside wall; (b) $a / b=0.30$, the $\mathrm{TM}_{01}$ and the $\mathrm{TM}_{11}$ modes are confined by the PBG structure [9]. be made of absorber or be located relatively far from the center of the PBG structure. For $a / b>0.2$ the PBG structure confines the higher-order $\mathrm{TM}_{11}$-like mode [Fig. 3(b)], and is no longer attractive for accelerators, since the $\mathrm{TM}_{11}$ mode is a dangerous wakefield mode. The electric field of the $\mathrm{TM}_{11}$ mode has a transverse component, and thus, the dipole mode is primarily responsible for kicking the electron beam off axis. Thus, only PBG structures with $0.1<$ $a / b<0.2$ can be applied for the construction of a singlemode accelerator cavity.

\section{2D PBG RESONATOR CONSTRUCTION AND TESTING}

\section{A. Brass PBG resonator testing}

In order to verify experimentally the mode confinement and higher-order mode suppression in PBG resonators, we constructed two PBG resonators for cold testing (Fig. 4). The resonators were fabricated using brass cylinders closed at each end by brass circular plates. PBG structures were formed by brass rods fitted into arrays of holes at the end plates. A single rod was missing from the center of the PBG structure to form the PBG resonator. Dimensions of the resonators are summarized in Table I. In cavity 1 , with $a / b<0.2$, only the $\mathrm{TM}_{01}$ mode was confined by the PBG

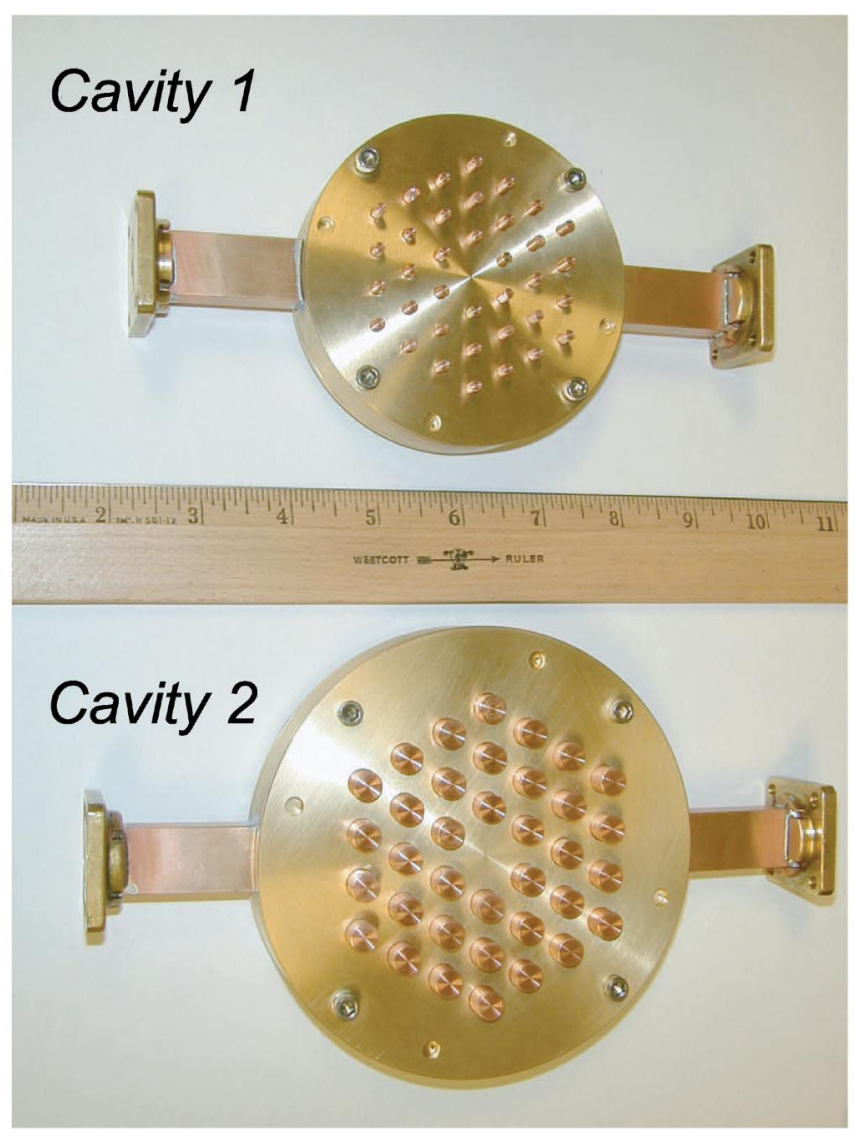

FIG. 4. (Color) PBG resonators built for the cold test. 
TABLE I. The parameters of two PBG cavities constructed for the cold test.

\begin{tabular}{lcc}
\hline \hline \multicolumn{1}{c}{ Parameter } & Cavity 1 & Cavity 2 \\
\hline Lattice vector, $b$ & $1.06 \mathrm{~cm}$ & $1.35 \mathrm{~cm}$ \\
Rod radius, $a$ & $0.16 \mathrm{~cm}$ & $0.4 \mathrm{~cm}$ \\
$a / b$ & 0.15 & 0.3 \\
Cavity radius & $3.81 \mathrm{~cm}$ & $4.83 \mathrm{~cm}$ \\
Eigenfrequency of the $\mathrm{TM}_{01}$ mode & $11 \mathrm{GHz}$ & $11 \mathrm{GHz}$ \\
Eigenfrequency of the $\mathrm{TM}_{11}$ mode & $15.28 \mathrm{GHz}$ & $17.34 \mathrm{GHz}$ \\
Axial length of the cavity & $0.787 \mathrm{~cm}$ & $0.787 \mathrm{~cm}$ \\
\hline \hline
\end{tabular}

structure and in the cavity 2 , with $a / b=0.3$ and thus $a / b>0.2$, the $\mathrm{TM}_{01}$ and the $\mathrm{TM}_{11}$ modes were confined. Cavities 1 and 2 were designed so that the eigenfrequencies of the $\mathrm{TM}_{01}$ modes were the same, at $11 \mathrm{GHz}$. This frequency was chosen for convenience of fabrication and to maintain the frequency of the dipole mode below $18 \mathrm{GHz}$, the limit of the available calibration kits for the network analyzer. WR62 waveguides were employed to feed rf power into the resonators, and the same size waveguides were connected symmetrically on the opposite sides.

To couple the power into a PBG resonator several rods in front of the waveguide aperture were removed. Several coupling schemes were tried. The coupling scheme finally used in all experiments is shown in Fig. 5. The removed rods are shown in Fig. 5 with hollow circles. The PBG structure rods are shown with filled circles. We measured the $S_{21}$ (transmission) elements of the scattering matrix using the HP8510 vector network analyzer. First measurements were performed without placing eccosorb at the outside metal walls of the cavities and thus the eigenmodes of the central PBG resonator as well as the eigenmodes of

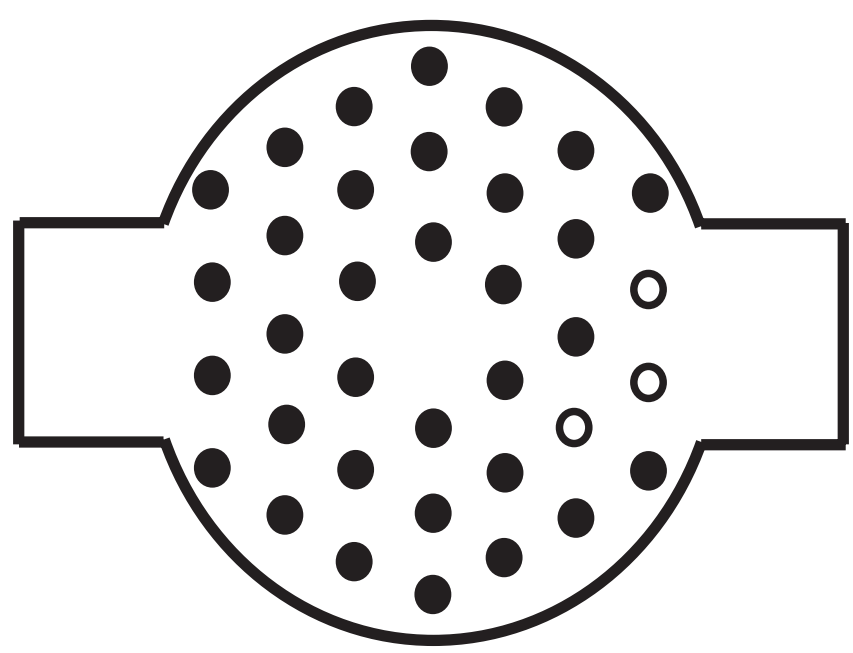

FIG. 5. Coupling scheme for the PBG resonator. PBG structure rods are shown with filled circles. Missing rods are shown with hollow circles. the pillbox resonator as a whole, formed by the outside metal wall, were confined. The coupling curves for both resonators are shown in Fig. 6 (upper graphs). Next, a new set of measurements was performed with eccosorb placed along the outside metal wall of the cavities. The transmission curves for the cavities with eccosorb are shown in Fig. 6, lower graphs. It can be seen from the figure that the higher-order resonances in cavity 1 (from 14 to $17 \mathrm{GHz}$ ) have broadened and shifted to higher frequencies. This proves that those resonances are not confined inside the defect of the PBG structure, but instead are supported by the metal wall of the cavity. According to our estimates, the eccosorb reduced the $Q$ factors of the modes not confined by the PBG structure by a factor of 10 . The $Q$ factor and the frequency of the $\mathrm{TM}_{01}$ mode at $11 \mathrm{GHz}$ did not change in cavity 1 . The $Q$ factor and the frequency of both, the $\mathrm{TM}_{01}$ mode at $11 \mathrm{GHz}$ and the $\mathrm{TM}_{11}$ mode at $17 \mathrm{GHz}$, did not change in cavity 2 because of thicker rods and a wider band gap. This proves that those modes are confined inside the defect of the PBG structure, in agreement with the theoretical design of the resonators, as described in Sec. II. The Ohmic $Q$ factors for the $\mathrm{TM}_{01}$ modes in both cavities were measured of about 2000 .

\section{B. High $Q$ PBG resonator fabrication and testing}

Although the first cold tests successfully demonstrated the confinement of modes in PBG resonators, we observed a big discrepancy between the measured $Q$ factor of the confined modes, about 2000, and the theoretically calculated $Q_{w}$ of 5500. We suspected that the reason for the low $Q_{w}$ was the poor electrical contact between the rods and the end plates. The currents flowing from the rods onto the
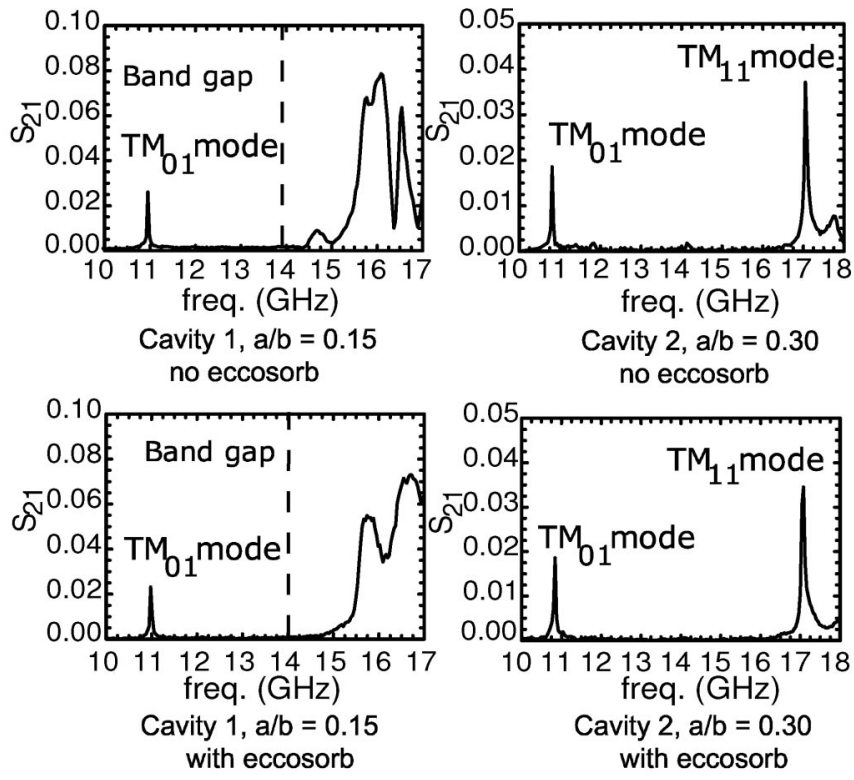

FIG. 6. Cold test measurements of the transmission coefficient $S_{21}$ for the brass resonators cavity 1 and cavity 2 . 


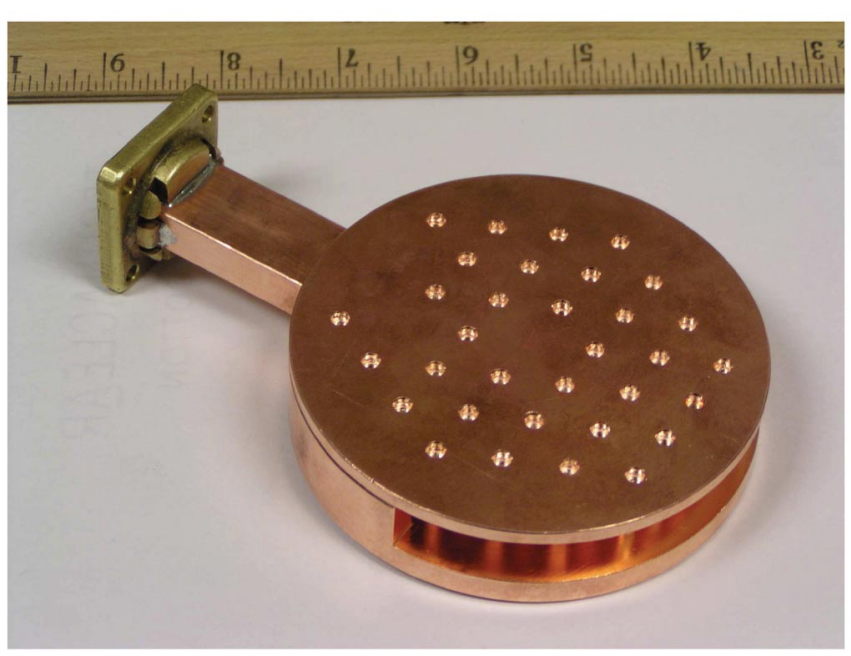

FIG. 7. (Color) Brazed PBG resonator.

plates were broken at the gaps in between rods and plates and large losses occurred. To improve the connection between rods and plates and decrease losses, brazing was performed. A new resonator with the same dimensions as cavity 1 was fabricated. Rods and plates were now machined of high purity oxygen-free class 1 copper. Brazing material was placed into the gaps between rods and plates and then the structure was heated in a hydrogen furnace. Good electrical connection was made. The brazed resonator (Fig. 7) was tested and the $Q$ factor of 5000 was measured for the $\mathrm{TM}_{01}$ mode. This is in excellent agreement with theory.

In addition to brazing, one more improvement was made to the resonator design. Half of the outer wall was removed to obtain the HOM damping (visible in Fig. 7). The other half of the wall remained in place since it is required to obtain good input coupling. Since the measured $Q$ was high, we concluded that the removal of the wall did not affect the $\mathrm{TM}_{01}$ mode confinement. This is in agreement with theory. Removing a part of the wall also allowed us to tune the frequency of the $\mathrm{TM}_{01}$ mode by deforming the rods of the inner row. A fishing line was wrapped around the inner row rod and pulled, and the rod was slightly bent. As a result of the deformation, the frequency of the $\mathrm{TM}_{01}$ mode was tuned from 11.010 to $11.000 \mathrm{GHz}$.

\section{PBG ACCELERATOR DESIGN}

The successful construction of several PBG resonators proving the mode confinement in PBG structures and HOM damping, formed the basis to proceed with demonstration of a PBG accelerating structure [9]. The accelerating structure schematic in the form of a disk-loaded PBG waveguide is shown in Fig. 8. The PBG waveguide was formed by removing the central rod in a triangular array of metal rods. The complete design of the traveling-wave PBG accelerator must determine the following parameters:

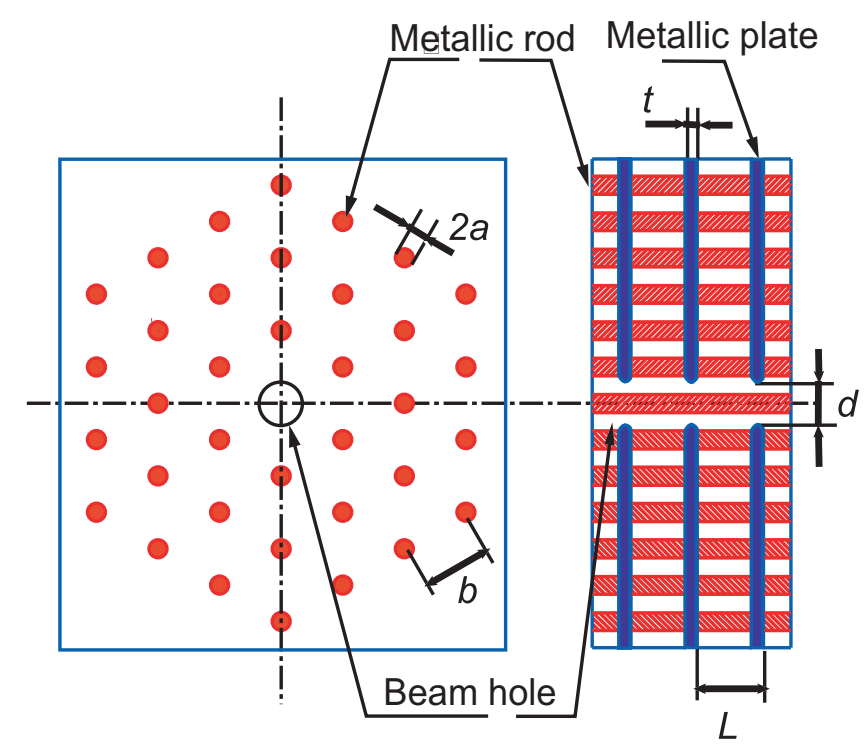

Front view

Side view

FIG. 8. (Color) Disk-loaded PBG waveguide [9].

the rod radius $a$, the spacing between the rods $b$, the plate spacing $L$, the optimal phase shift per cell $\varphi$, the iris thickness $t$, and the iris round beam opening diameter $d$ (see Fig. 8). In addition, the coupler cell must be designed separately. The coupling scheme (number of removed rods) must be chosen first. Next, the rod radius and the spacing between the rods must be determined separately for the coupling cell.

The $\varphi=2 \pi / 3$ phase shift per cell was chosen for the accelerator structure. The requirement of the resonance between the beam and the electromagnetic wave determined the plate spacing, $L=5.83 \mathrm{~mm}$ :

$$
\frac{\omega L}{c}=\frac{2 \pi}{3},
$$

where $\omega=2 \pi f, f=17.140 \mathrm{GHz}$ is the accelerator frequency, and $c$ is the speed of light. The iris plate beam opening diameter, $d=4.32 \mathrm{~mm}$, was determined by the compatibility of the structure with the waist size of the electron beam produced at the Haimson/MIT accelerator laboratory [11]. The iris thickness $t=1.14 \mathrm{~mm}$ was chosen to achieve high shunt impedance, based on the requirement that a 6-cell structure had to accelerate the electron beam by more than $1 \mathrm{MeV}$ for $2 \mathrm{MW}$ of input power available from the klystron. Finally, the radius of the rod, $a$, and the spacing between the rods, $b$, had to be adjusted to bring the cell's frequency to $17.140 \mathrm{GHz}$. Each parameter, $a$ and $b$, could be varied to change the frequency. However, it was desirable to keep the ratio $a / b \approx$ 0.15 for good HOM damping and high Ohmic $Q$. The exact values of $a$ and $b$ were determined after the coupling cell was tuned. First, $a$ and $b$ of the coupler cell were chosen for the optimal coupling. Next, the spacing between the rods $b$ 


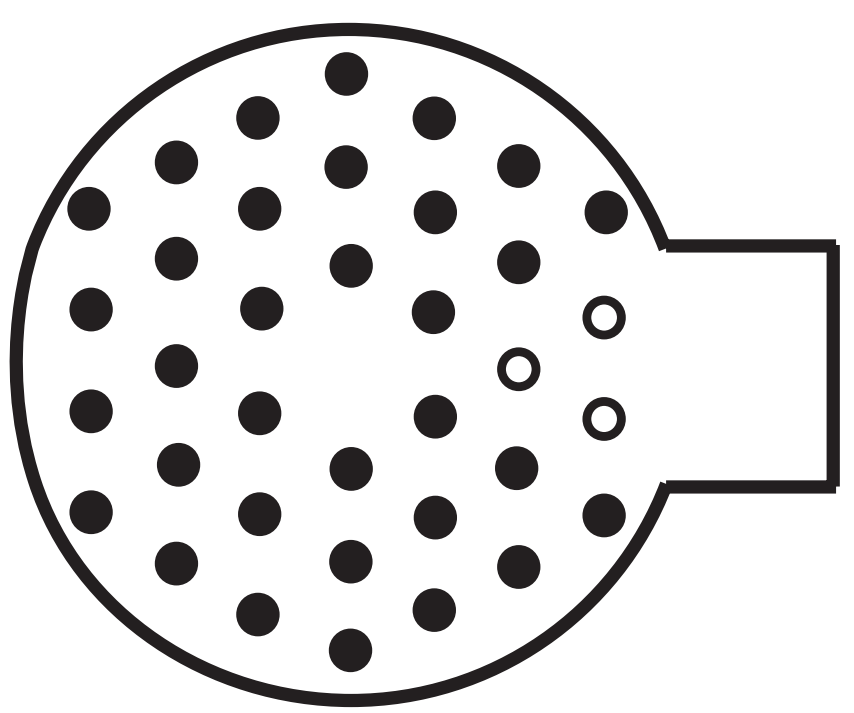

FIG. 9. Coupling scheme for the PBG coupler of a travelingwave PBG accelerator. PBG structure rods are shown with filled circles. Missing rods are shown with hollow circles.

was fixed for all the TW cells and the coupling cells. The rod radius, $a$, was determined for the traveling-wave cells to achieve the correct frequency of $17.140 \mathrm{GHz}$.

HFSS was applied to design the PBG coupler. We employed the periodic voltage standing-wave ratio method $[12,13]$. First, the coupling scheme was chosen with 3 rods removed from the PBG structure (Fig. 9). The PBG diskloaded structure was replaced with the cylindrical diskloaded waveguide with the same dispersion to simplify the computations. Then we wrote a $\mathrm{C}++$ code driving HFSS to optimize the rod radius and the spacing in the coupler cell. The reflection from the coupler cell was computed according to $[12,13]$ and the rod radius and spacing were varied until the coupling with a low reflection of $|R|=0.012$ was achieved. The optimized coupler parameters were found as follows: the rod radius $a=1.05 \mathrm{~mm}$, the distance between the rods $b=6.97 \mathrm{~mm}$.

To complete the design of a PBG accelerator we tuned the rod radius in the TW PBG cells with the rod spacing of $b=6.97 \mathrm{~mm}$ so that at $2 \pi / 3$ phase shift the cells had the frequency of $17.140 \mathrm{GHz}$. It was found that for the rod radius $a=1.04 \mathrm{~mm}$, the cell's frequency was correct. The ratio of $a / b$ in the newly designed cell was found to be 0.149. The electric field magnitude in the resulting PBG disk-loaded waveguide is shown in Fig. 10. The dimensions of the PBG accelerator structure are summarized in Table II [9]. Table II also presents the computed accelerator characteristics of the designed PBG accelerator structure, including the structure Ohmic $Q$ factor, $Q_{w}$, the shunt impedance per unit length

$$
r_{s}=\frac{\left(\int_{0}^{L} \vec{E} \cdot d \vec{s}\right)^{2}}{P_{w} L},
$$

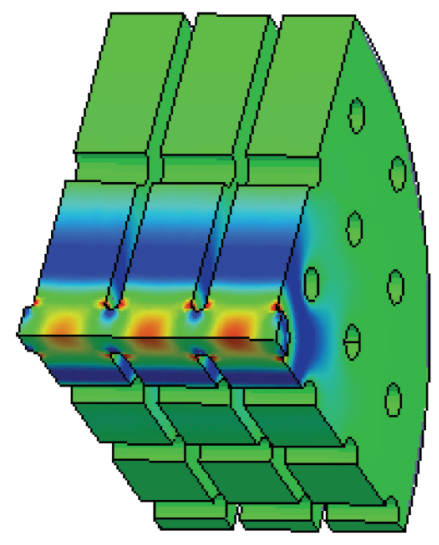

FIG. 10. (Color) Magnitude of the electric field distribution in a PBG disk-loaded waveguide. One quarter of the PBG waveguide is shown.

the group velocity, and the gradient

$$
G=\frac{\int_{0}^{L} \vec{E} \cdot d \vec{s}}{L}
$$

In formulas (2) and (3) the electric field $\vec{E}$ is integrated along the axis of the accelerating cavity of the length $L, P_{w}$ is the electromagnetic power lost in the walls of the cavity due to Ohmic heating.

Finally, the tolerances were studied. The dependence of $|R|$ on $a$ and $b$, was calculated (Fig. 11). It can be seen from the picture that a coupler, which has either rod dimensions or the spacing between the rods different from the original by $0.025 \mathrm{~mm}(0.001 \mathrm{in}$.) has $|R| \sim 0.6$. This means that the tuning of the coupler is likely to be needed after fabrication with standard manufacturing tolerance. The dependence of the TW cell frequency on the dimension variance is shown in Fig. 12. It can be seen from the graph that $0.025 \mathrm{~mm}$ (0.001 in.) deviation in either rod radius or spacing produces almost $100 \mathrm{MHz}$ frequency shift. Thus, cell tuning is likely to be required after conventional fabrication.

TABLE II. The dimensions and accelerator characteristics of the PBG accelerator structure [9].

\begin{tabular}{lc}
\hline \hline Rod radius (TW cell/coupler cell), $a$ & $1.04 \mathrm{~mm} / 1.05 \mathrm{~mm}$ \\
Spacing between the rods, $b$ & $6.97 \mathrm{~mm}$ \\
$a / b$ & 0.149 \\
Plates spacing, $L$ & $5.83 \mathrm{~mm}$ \\
Phase advance per cell & $2 \pi / 3$ \\
Iris radius, $d / 2$ & $2.16 \mathrm{~mm}$ \\
Iris thickness, $t$ & $1.14 \mathrm{~mm}$ \\
Frequency $\left(\mathrm{TM}_{01}\right.$ mode) & $17.140 \mathrm{GHz}$ \\
Ohmic $Q$ factor, $Q_{w}$ & 4188 \\
Shunt Impedance, $r_{s}$ & $98 \mathrm{M} \Omega / \mathrm{m}$ \\
{$\left[r_{s} / Q_{w}\right]$} & $23.4 \mathrm{k} \Omega / \mathrm{m}$ \\
Group velocity & $0.013 c$ \\
Gradient & $25.2 \sqrt{P[\mathrm{MW}]} \mathrm{MV} / \mathrm{m}$ \\
\hline
\end{tabular}




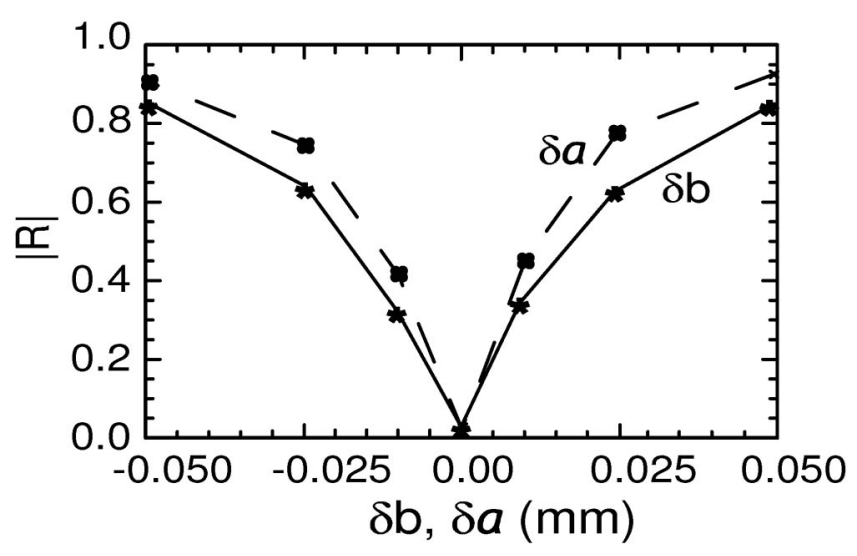

FIG. 11. The dependence of the reflection from the coupler on the deviation of the rod radius $a$ and the spacing between the rods $b$ from the optimized dimensions.

\section{PBG ACCELERATOR CONSTRUCTION AND COLD TESTS}

Two PBG accelerator structures were fabricated. For the first structure, the rods and plates were machined out of class 1 oxygen-free electronic copper. The plates were placed in slots of the side pieces, and the rods were mounted into the arrays of round holes in the plates. Although, brazing of a single-cell PBG resonator was successful, brazing of a multicell structure was determined to be too complicated using our available resources. Therefore, the structure was assembled by bolting the parts together. Without brazing, the Ohmic losses in the structure were found in cold test to be too high for good PBG accelerator performance. Hence, this approach was abandoned.

The second structure was fabricated via electroforming. Rods and plates were grown together to form a single copper part. Then a good electrical connection between rods and plates in the structure is automatically ensured. The aluminum mandrels with holes in place of the rods

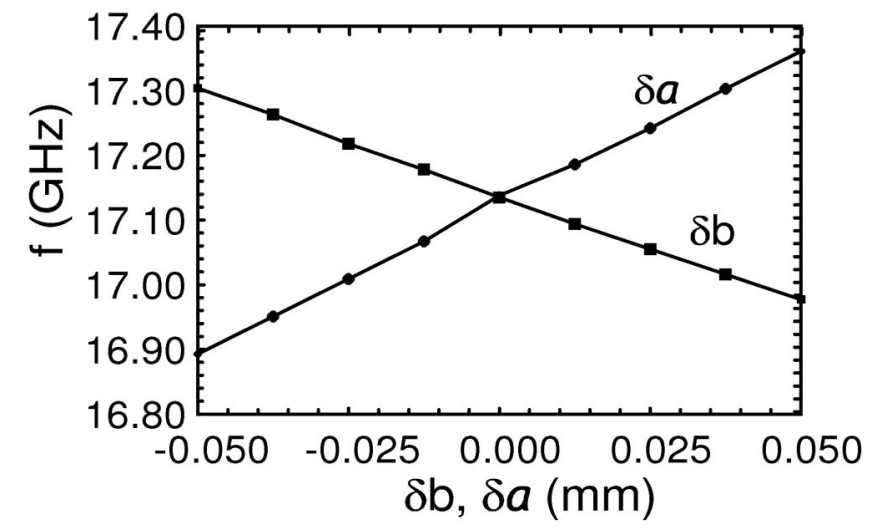

FIG. 12. The dependence of the TW cell frequency on the deviation of the rod radius $a$ and the spacing between the rods $b$ from the optimized dimensions. were fabricated, and then placed into copper solution. Copper ions were deposited on the mandrels, and PBG cells consisting of the rods and two half-plates were formed. Then the aluminum was etched away. Cells were put together and soldered. Copper was flashed over the whole structure to cover the solder in the joints. The waveguides were bolted to the structure. Side pieces were bolted for additional mechanical strength. A photograph of the electroformed structure is shown in Fig. 13.

The coupling curves for the new structure were measured with an HP8720 network analyzer. The comparison of the measured and computed reflection curves indicated that the fabricated structure was $40 \mathrm{MHz}$ higher in frequency than the design. This frequency discrepancy implied that the structure dimensions were within the standard manufacturing tolerance of $0.025 \mathrm{~mm}$ (0.001 in.). However, tuning was required, as predicted, to bring the structure to the correct frequency. In addition, the resonance frequencies of each TW cell were measured. It was found that two cells were $10 \mathrm{MHz}$ lower in frequency than the other four cells. So two cells had to be tuned differently.

Tuning was performed by chemical etching of the copper rods. The structure was mounted on a stainless steel rod with a length of about $12 \mathrm{in}$. The rod was wrapped with heat shrink tubing and tape to a diameter that provided a press fit into the beam hole of the structure and then was inserted into the beam hole. This served both to mask the beam hole and as a handle for the dipping operation. Jacko'-lantern candles were melted in a beaker on a hot plate and served as a masking material for two cells which were low in frequency. This wax was found to be suitably resistant to chemical attack by the acid solution. Acid solution was mixed as follows: $100 \mathrm{ml}$ nitric acid, $275 \mathrm{ml}$ phosphoric acid, $125 \mathrm{ml}$ acetic acid. This solution was used at $45^{\circ} \mathrm{C}$ and removed $0.0025 \mathrm{~mm}$ (0.0001 in.) per minute from each side of the copper rods. The whole structure was etched for $3 \mathrm{~min}$. Next, two cells with low frequencies were masked and the rest of the structure was
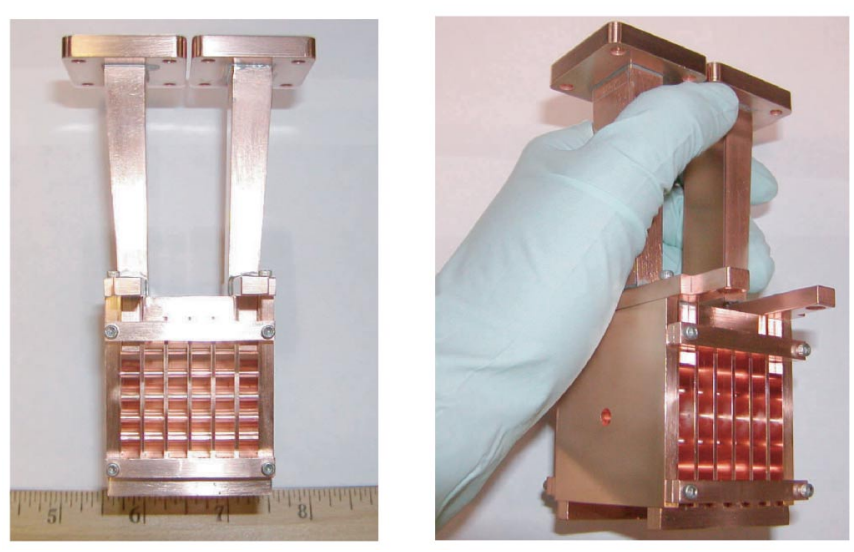

FIG. 13. (Color) Photographs of the electroformed PBG accelerator structure. 
etched for one more minute. Finally, the wax was removed with a detergent cleaner in an ultrasonic tank at $70^{\circ} \mathrm{C}$. The structure was rinsed with deionized water, dipped in chromic acid solution for final cleaning of tarnish spots from the previous steps, rinsed in deionized water again and dried.

The tuned structure was cold-tested again. The measured reflection $\left(S_{11}\right)$ and transmission $\left(S_{21}\right)$ curves are shown in Fig. 14 [9]. For comparison, the $S_{11}$ and $S_{21}$ curves computed with HFSS are shown. There is very good agreement between the measured and the computed curves. The reason for the slightly increased dispersion of the measured curves is the etching process. While the etching decreased the rod diameter it also decreased the plate thickness and thus increased the dispersion.

The field profile of the structure eigenmode at $17.140 \mathrm{GHz}$ was measured by a bead pull method. A $0.8 \mathrm{~mm}(1 / 32 \mathrm{in}$.$) diameter nylon (\varepsilon \sim 3)$ bead was mounted on a fishing line. The line with the bead was aligned along the structure axis and pulled though the beam hole with a motor. An HP8720 network analyzer was used to record the phase shift of the signal transmitted through the structure. The on-axis field profile was computed from the recorded phase shifts. The field profile was found to be flat (Fig. 15), and the electric field had about the same magnitude in all six cells.

Finally, the temperature dependence of the structure eigenfrequencies was studied. Powering the accelerator structure with several MW of rf may lead to structure heating due to Ohmic loss. Therefore it is important to know how the accelerating mode frequency will behave with temperature. The structure was placed inside an oven and heated slowly at about $5^{\circ} \mathrm{C} /$ hour. This ensured a uniform temperature throughout the structure. The frequency of the accelerating mode was recorded. The data show that the eigenmode frequency decreases with temperature at the rate of about $1 \mathrm{MHz}$ per $5^{\circ} \mathrm{C}$. To estimate the temperature rise due to microwave heating of the PBG accelerator structure the following formula was applied $[14,15]$ :

$$
\Delta T=R_{s} H_{\mathrm{rod}}^{2} \sqrt{\frac{t_{\mathrm{pulse}}}{\pi \rho c_{v} k}},
$$

where $R_{s}$ is the structure shunt impedance, $H_{\text {rod }}$ is the maximum magnetic field on the rod, $t_{\text {pulse }}$ is the microwave pulse length, $\rho=8900\left(\mathrm{~kg} / \mathrm{m}^{3}\right)$ is the copper density, $c_{v}=385\left(\mathrm{~J} / \mathrm{kg}^{0} \mathrm{C}\right)$ is the specific heat per unit mass of the copper, $k=380\left[\mathrm{~W} /\left({ }^{\circ} \mathrm{C} \cdot \mathrm{m}\right)\right]$ is the thermal conductivity of copper. For the PBG accelerator with the average gradient of $30 \mathrm{MV} / \mathrm{m}$ and $100 \mathrm{~ns}$ pulse the temperature rise will not exceed $5{ }^{\circ} \mathrm{C}$, which means that the frequency shift for the accelerating mode will not exceed $1 \mathrm{MHz}$. The temperature rise mostly happens at the first row of rods and is greater by a factor of 2 than in a regular disk-loaded waveguide $[15,16]$. In future practical realizations of a
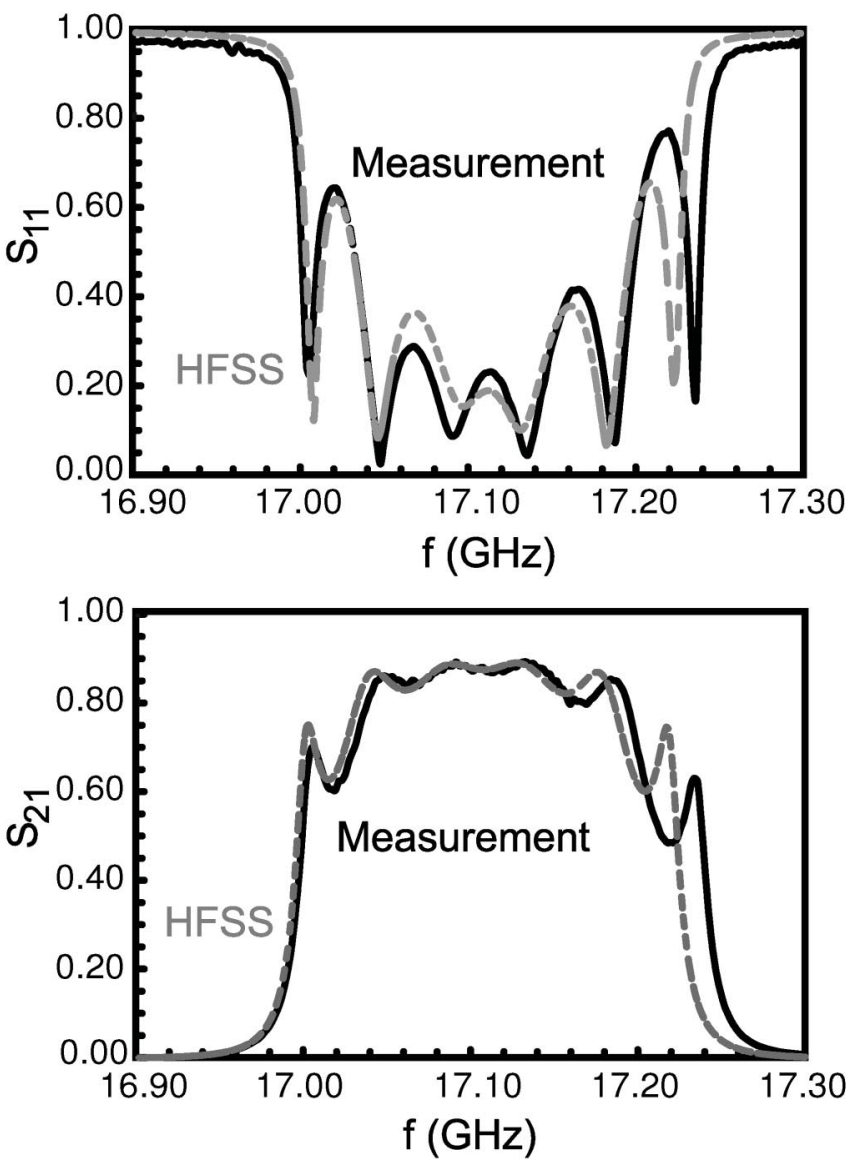

FIG. 14. Comparison between measured and computed $S_{11}$ and $S_{21}$ coupling curves for the tuned PBG accelerator structure [9].

PBG accelerator the first row of rods in the PBG structure should be water cooled to dissipate the heat. The structure should also be optimized to minimize the temperature rise due to the microwave pulsed heating and to avoid reaching the damage threshold.

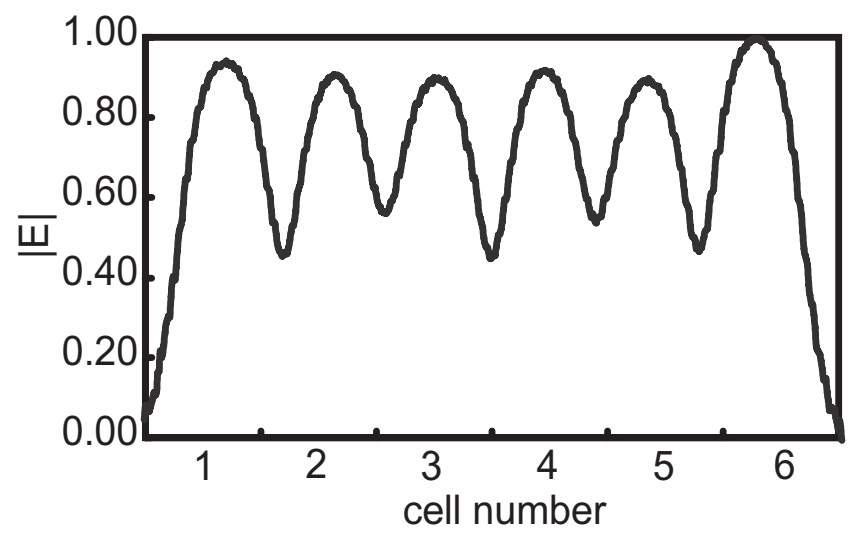

FIG. 15. Field profile of the accelerating mode for the tuned PBG accelerator structure. 


\section{CONCLUSION}

This work was performed to construct the first travelingwave linear photonic band gap accelerator structure. The advantage of the PBG accelerator over the conventional disk-loaded accelerator is the efficient long-range wakefields suppression. Suppression of wakefields would allow scientists to extend the operating frequencies of future linear colliders to higher frequencies without sacrificing beam quality.

First, 2D PBG resonators were studied and the design of a resonator which would completely suppress the wakefields was presented. Numerous 2D PBG resonators were constructed and tested. Two goals were achieved: the 2D metallic PBG structures theory and simulations were benchmarked, and single-mode confinement in a PBG resonator was proven. Engineering efforts were undertaken to successfully demonstrate a high $Q$ PBG resonator. Brazing was used to provide electrical contact between metallic rods and plates.

Second, the first traveling-wave PBG accelerator structure was designed. The structure was modeled with HFSS. The first PBG coupler with excellent mode symmetry was designed. Computational techniques for PBG coupler tuning were developed. A total of two TW PBG structures were constructed and cold tested. The first structure was bolted and although it proved the correctness of the computational design, it was not appropriate for hot test, because of high Ohmic losses. The second structure was electroformed. The electroforming proved to be a good technique for fabrication of multicell PBG structures. The tuning of the PBG accelerator structure was performed via etching. The cold tests of the tuned structure were in perfect agreement with computations.

The TW PBG structure was finally installed at the MIT accelerator laboratory for hot testing. The results of the hot test are described in [9]. The structure was powered with 2 MW of microwaves. The acceleration of an electron beam inside the PBG accelerator structure was measured. It was found that the beam energy increased as the square root of the input power as expected from theory. Maximum energy gain for $2 \mathrm{MW}$ of input power was found to be 1.4 $\mathrm{MeV}$ which is consistent with the estimated $35 \mathrm{MV} / \mathrm{m}$ accelerating gradient [9]. Further testing, including a hot test demonstration of wakefield suppression inside the PBG accelerator, is being planned.

\section{ACKNOWLEDGMENTS}

This work was supported by the Department of Energy, Division of High Energy Physics Contract No. DE-FG02-
91ER40648. The authors wish to thank Monica Blank and Philipp Borchard of CPI for brazing the PBG resonator and Clency Lee-Yow of Custom Microwave for electroforming the traveling-wave PBG structure. The authors also acknowledge very helpful discussions with Chiping Chen, Valery Dolgashev, Amit Kesar, Frank Krawczyk, Warren K. Pierce, James M. Potter, and Thomas Wangler.

[1] J. D. Joannopoulos, R. D. Meade, and J. N. Winn, Photonic Crystals: Molding the Flow of Light (Princeton University Press, Princeton, 1995).

[2] D. R. Smith, S. Schultz, N. Kroll, M. Sigalas, K. M. Ho, and C. M. Souloulis, Appl. Phys. Lett. 65, 645 (1994).

[3] M. A. Shapiro, W. J. Brown, I. Mastovsky, J. R. Sirigiri, and R. J. Temkin, Phys. Rev. ST Accel. Beams 4, 042001 (2001).

[4] C. Adolphsen, K. Bane, T. Higo, K. Kubo, R. Miller, R. Ruth, K. Thompson, and J. Wang, Phys. Rev. Lett. 74, 2475 (1995).

[5] K.L.F. Bane and Z. Li, Stanford Linear Accelerator Center Report No. SLAC-PUB-8545, 2000 (unpublished).

[6] E. Yablonovitch, Phys. Rev. Lett. 58, 2059 (1987).

[7] E. I. Smirnova, C. Chen, M. A. Shapiro, J. R. Sirigiri, and R. J. Temkin, J. Appl. Phys. 91, 960 (2002).

[8] D. R. Smith, D. Li, D. C. Vier, N. Kroll, and S. Schultz, in Advanced Accelerator Concepts: Seventh Workshop, edited by Swapan Chattopadhyay, Julie McCullough, and Per Dahl, AIP Conf. Proc. No. 398 (AIP, New York, 1997), p. 518.

[9] E. I. Smirnova, A. S. Kesar, I. Mastovsky, M. A. Shapiro, and R. J. Temkin, Phys. Rev. Lett., 95, 074801 (2005).

[10] High Frequency Structure Simulator, www.hfss.com, Ansoft Corporation, Pittsburgh, PA 15219.

[11] J. Haimson and B. Mecklenburg, in Proceedings of the 1995 Particle Accelerator Conference (IEEE, Piscataway, NJ, 1995), Vol. 2, p. 755.

[12] N. M. Kroll, C. K. Ng, and D. C. Vier, Stanford Linear Accelerator Center Report No. SLAC-PUB-8614, 2000 (unpublished).

[13] C. Nantista, S. Tantawi, and V. Dolgashev, Phys. Rev. ST Accel. Beams 7, 072001 (2004).

[14] P. B. Wilson, Stanford Linear Accelerator Center Report No. SLAC-PUB-7449, 1997 (unpublished).

[15] Z. Li, N. T. Folwell, K. Ko, R. J. Loewen, E. W. Lundahl, B. McCandless, R. H. Miller, R. D. Ruth, M. D. Starkey, Y. Sun, and J. W. Wang, Stanford Linear Accelerator Center Report No. SLAC-PUB-8647, 2000 (unpublished).

[16] M. A. Shapiro, W. J. Brown, C. Chen, J. R. Sirigiri, E. I. Smirnova, and R. J. Temkin, in Proceedings of the 2001 Particle Accelerator Conference, edited by P. Lucas and S. Webber (IEEE, Piscataway, NJ, 2001), Vol. 2, p. 930. 